\title{
Frequency Threshold for Ablation During Holmium Laser Lithotripsy: How High Can You Go?
}

\author{
Ali H. Aldoukhi, MD, MS, ${ }^{1}$ Kristian M. Black, MD, MS, ${ }^{1}$ Timothy L. Hall, PhD, \\ William W. Roberts, MD, ${ }^{1,2}$ and Khurshid R. Ghani, MD, MS
}

\begin{abstract}
Purpose: We performed in vitro studies to assess the relationship of pulse frequency on stone ablation during contact laser lithotripsy and determine if there is a threshold after which its effect on lithotripsy is limited.

Methods: BegoStones were fragmented using a Ho:YAG laser (P120 Moses) and a $230 \mu \mathrm{m}$ fiber at $0.5 \mathrm{~J}$ on long pulse (LP) and Moses distance (MD) modes in contact with the stone. The relationship between the number of pulses $(1-40 \mathrm{~Hz})$ on stone crater volume was assessed using three-dimensional confocal microscopy and nonlinear-segmented regression. To simulate a painting technique, we assessed fragmentation (mg/second) at 20,40 , and $60 \mathrm{~Hz}$, with the fiber moving at a speed of 1 and $3 \mathrm{~mm} /$ second, respectively. High-speed imaging was used to record ablation.

Results: When the laser fiber was fixed, after 13.0 (LP) and 15.4 (MD) pulses, greater pulse frequency did not lead to a significant increase in stone crater volume. Fragmentation was greatest at higher frequencies and faster fiber speed. Increasing the frequency from 20 to $60 \mathrm{~Hz}$ at $3 \mathrm{~mm} / \mathrm{second}$ increased fragmentation by $82 \%$ and $61 \%$ for LP and MD modes, respectively. Using high-speed data, if the laser fiber is moving at $1 \mathrm{~mm} / \mathrm{second}$, a hypothetical frequency threshold for ablation was calculated to be 52 and $61.6 \mathrm{~Hz}$ for LP and MD modes, respectively.

Conclusion: Increasing the fiber speed increases stone ablation when using high frequency settings. When the fiber is fixed there is a threshold after which increasing the pulse frequency leads to minimal gain in ablation. The exact value for threshold when the fiber is moving needs further study. Our study serves to provide insight for parameter selection and safety of laser lithotripsy for dusting technique.
\end{abstract}

Keywords: kidney stone, holmium laser, frequency, dusting, lithotripsy, Moses

\section{Introduction}

$\mathbf{F}$ IRST-GENERATION HOLMiUm laser systems were lowpower machines with pulse frequency limited to 15 to $20 \mathrm{~Hz}$. Next-generation holmium lasers provide urologists with higher power, and frequencies up to $80 \mathrm{~Hz} .{ }^{1}$ Higher frequencies have led to increasing interest in dusting techniques that use low pulse energy and high frequency settings to break stones into fine fragments and powder. ${ }^{2,3}$ Although increasing the pulse energy increases fragmentation regardless of the frequency applied, ${ }^{4-6}$ it comes at the expense of greater stone retropulsion, larger fragment sizes, and increased fiber tip degradation. ${ }^{7}$ In contrast, the effect of increasing pulse frequency on fragmentation is less clear.

Prior study on understanding the effect of pulse frequency on fragmentation may suggest that there is a threshold for pulse frequency. ${ }^{4,5,8}$ Frequencies below the threshold result in greater fragmentation, but frequencies above the threshold may result in minimal increase in fragmentation. In an early study, a significant increase in fragmentation was noted when the frequency increased from 5 to $10 \mathrm{~Hz}$ when using a pulse energy of 0.5 and $1 \mathrm{~J}$, but not when increased from 10 to $15 \mathrm{~Hz} .{ }^{8}$ Similarly, another in vitro study reported that there was a minimal increase in fragmentation volume when pulse frequency was increased. ${ }^{4}$ In contrast, a recent study by Sea and colleagues found that fragmentation increased significantly when pulse frequency increased from 10 to $40 \mathrm{~Hz}$ using pulse energy of $0.2 \mathrm{~J} .^{5}$ However, no studies to date take into account how the frequency threshold may be affected by the technique used during lithotripsy, and in particular the speed at which the laser fiber is moving.

Departments of ${ }^{1}$ Urology and ${ }^{2}$ Biomedical Engineering, University of Michigan, Ann Arbor, Michigan, USA.

A video demonstrating this technique is available online. 
The aim of this experimental in vitro study was to investigate the effect of pulse frequency on stone fragmentation at a fixed location, as well as pulse frequency with the laser fiber moving at different speeds. In addition, the effect of frequency when using pulse modulation with the Moses $^{\mathrm{TM}}$ Technology was studied. Furthermore, since frequency impacts the total power applied to the stone and surrounding fluid, efforts to understand a threshold for frequency settings can have implications for improving the safety of laser lithotripsy.

\section{Methods}

\section{Experimental setup}

An artificial BegoStone model $(30 \times 30 \times 3 \mathrm{~mm}$, composition of 15:5) was made with a flat surface using an injection mold to ensure the stones were completely horizontal. A 120 W Moses holmium laser (Moses P120; Lumenis) and a $230 \mu \mathrm{m}$ core laser fiber (Moses $200 \mathrm{D} / \mathrm{F} / \mathrm{L}$; Lumenis) were used for lithotripsy; core fiber size was reported as stated in the package insert. All experiments were performed with pulse energy of $0.5 \mathrm{~J}$ utilizing long pulse (LP) and Moses distance (MD) modes; these pulse modes were selected because they are commonly used during dusting technique. The laser fiber was attached to a three-dimensional (3D) positioner system (Velmex). Furthermore, all experiments were conducted in water bath of room temperature deionized water.

\section{Pulse frequency threshold: fixed laser fiber experiments}

To assess the effect of increasing pulse frequency, when the laser fiber is fixed in contact with the stone, single pulses of $0.5 \mathrm{~J}$ were delivered to provide a total number of $1,5,10$, $15,20,25,30,35$, and 40 pulses at different locations on the stone, respectively. Each experiment was repeated five times resulting in a total of 45 trials. To deliver the exact number of pulses for each experiment, singles pulses were delivered by setting the system to the lowest possible frequency $(5 \mathrm{~Hz})$ with a quick step on the foot switch. High-speed videos were captured with high-speed camera (Fastcam SA1.1; Photron, Tokyo, Japan) at 10,000 frames per second to ensure only a single pulse was delivered each time, and confirm the exact delivery of the stated number of pulses for each experiment.

The laser fiber was initially placed in contact with the stone before the first pulse and was not moved for the remainder of the experiment. The laser fiber was stripped and cleaved with a scribe pen before each experiment. This was done to ensure optimal power delivery from the fiber to the stone. ${ }^{9,10}$ Outcomes were crater depth, area at the stone surface, and ablation volume, all measured using a 3D laser confocal microscope (LEXT OLS 4000; Olympus, Tokyo, Japan). In addition, high-speed videos were recorded to demonstrate ablation during the $1 \mathrm{st}, 5 \mathrm{th}, 10 \mathrm{th}, 20 \mathrm{th}, 30 \mathrm{th}$, and 40th pulse at the same stone location when delivering a total of 40 pulses using LP mode.

\section{Frequency threshold: moving laser fiber experiments}

To assess the effect of frequency and laser fiber movement on fragmentation, the fiber was advanced with the 3D positioning system during lithotripsy. The 3D positioner was programed using Matlab (MathWorks) to move the fiber and fragment the stone in a standardized manner of 10 lines. Each line was $20 \mathrm{~mm}$ long with $2 \mathrm{~mm}$ spacing between each line (Supplementary Fig. S1). The fiber was positioned in contact with the stone surface for the first line and repositioned before each line to account for fiber tip degradation and ensure consistent fiber to stone distance. This method was chosen to simulate a "painting" technique during dusting laser lithotripsy. ${ }^{11}$ Three different frequencies were assessed: 20, 40, and $60 \mathrm{~Hz}$. In addition, the positioner was programmed to move the fiber to cover the same stone distance at two different fiber speeds of 1 and $3 \mathrm{~mm} / \mathrm{second}$, equivalent to 3.3 minutes (200 seconds) and 1.1 minutes (67 seconds) of laser activation time, respectively.

Experiments were conducted in a water bath filled with $2 \mathrm{~L}$ of deionized water to control temperature change in the experimental environment; temperature change was found to be associated with fluid volume in the experimental model. ${ }^{12}$ Each experiment was repeated five times resulting in a total of 30 trials. The stones were weighed dry before overnight hydration and were left to desiccate for at least 24 hours after each experiment. The outcome was fragmentation rate, which was defined as the amount of mass lost over time (mg/second). In addition, videos demonstrating the number of pulses delivered at each stone location when the fiber is moving at $1 \mathrm{~mm} / \mathrm{second}$ were recorded.

\section{Statistical analysis}

Student's $t$-test was used to compare cohorts mean. All testing was two-sided and the probability of a type I error was set at 0.05. A nonlinear-segmented regression model with quadratic-plateau was used to determine the frequency threshold for crater ablation depth and volume, to estimate the number of pulses after which there was no statistically significant increase in stone fragmentation (SAS v9.4, Cary, NC).

\section{Results}

\section{Pulse frequency threshold: fixed laser fiber experiments}

Stone crater depth and volume increased with increasing the frequency until it reached a plateau after 15 pulses for both LP and MD pulse modes as demonstrated in Figure 1A and $\mathrm{B}$, respectively. Nonlinear-segmented model analysis (Fig. 2) estimated the ablation threshold for LP mode to be 14.8 and 13.0 pulses, for crater depth and volume, respectively. After this, increasing the frequency did not significantly increase ablation. For MD mode, the threshold was found to be 16.9 and 15.4 pulses for crater depth and volume, respectively. Crater area reached a plateau after 5 pulses for both pulse modes with no significant increase thereafter as shown in Figure 1C.

When comparing LP with MD mode, craters were significantly deeper for MD mode at 20, 25, 30, 35, and 40 pulses $(p<0.05)$. There were no significant differences between the two pulse modes for crater area and overall volume. The accompanying high-speed imaging video (Supplementary Video S1) shows an example of fragmentation at different pulse frequencies. The highest amount of 

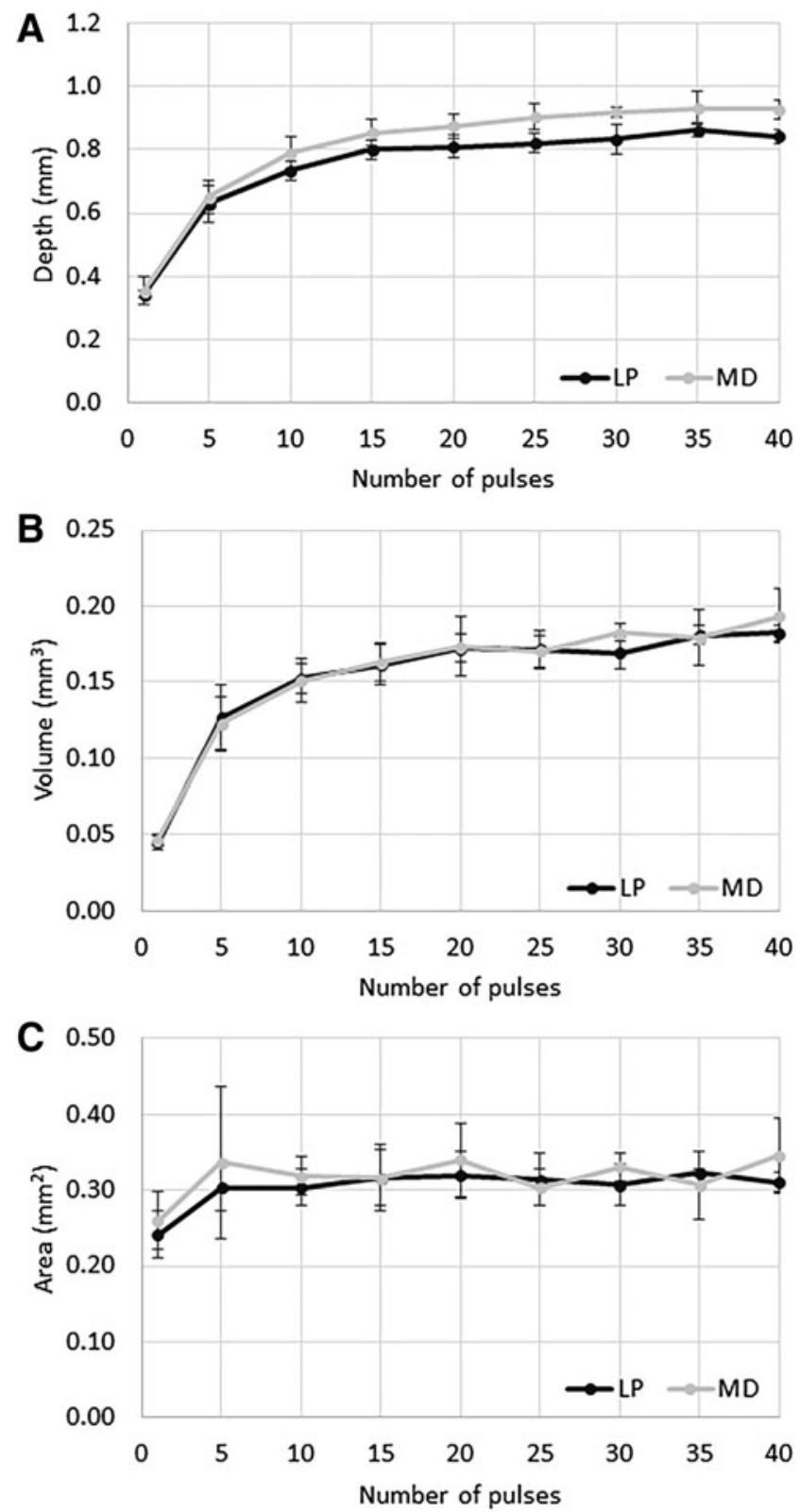

FIG. 1. Mean crater (A) depth, (B) volume, and (C) area at the stone surface for LP and MD modes at different number of laser pulses. $\mathrm{LP}=$ long pulse; $\mathrm{MD}=$ Moses distance.

dust was observed during the first pulse with less and less dust seen during the subsequent pulses.

\section{Frequency threshold: moving laser fiber experiments}

Figure 3 illustrates fragmentation rate when the fiber was moved at 1 and $3 \mathrm{~mm} / \mathrm{second}$ for LP and MD modes. Fragmentation rate increased with increasing pulse frequency for both fiber movement speeds. Moreover, fragmentation rate for all frequency settings and pulse modes increased significantly when the fiber moved at a speed of $3 \mathrm{~mm} / \mathrm{second}$ compared with $1 \mathrm{~mm} / \mathrm{second}$ (Fig. 3; $p<0.001$ ). Table 1 presents the percentage change in fragmentation for the different frequencies. The change was more pronounced when increasing frequency from 20 to $40 \mathrm{~Hz}$ than when increasing the frequency from 40 to $60 \mathrm{~Hz}$. In general, MD mode resulted in higher fragmentation rate than LP mode. The difference was statistically significant when using $60 \mathrm{~Hz}$ at fiber speed of $1 \mathrm{~mm} / \mathrm{second}$, and when using 20 and $40 \mathrm{~Hz}$ at fiber speed of $3 \mathrm{~mm} /$ second $(p<0.05)$. $p$-Values comparing fragmentation rate for MD to LP modes are provided in Supplementary Table S1. MD mode resulted in 7\%, 6\%, and 6\% more fragmentation at $1 \mathrm{~mm} /$ second compared with LP mode, when using 20,40, and $60 \mathrm{~Hz}$, respectively. Moreover, MD mode resulted in 22\%, $16 \%$ and $9 \%$ higher fragmentation at $3 \mathrm{~mm} / \mathrm{second}$ compared with LP mode, when using 20, 40 , and $60 \mathrm{~Hz}$, respectively.

High-speed imaging in Supplementary Video S2 demonstrates fiber movement of a $230 \mu \mathrm{m}$ fiber, and the number of pulses delivered over a $1 \mathrm{~mm}$ stone distance. At frequency of $20 \mathrm{~Hz}$ and fiber speed of $1 \mathrm{~mm} / \mathrm{second}$, there were $\sim 5$ pulses delivered at each distinct stone location. At $1 \mathrm{~mm} / \mathrm{second}$, the $230 \mu \mathrm{m}$ fiber strikes the stone in four distinct areas during the 1 second of laser activation (Fig. 4). For instructive purposes, it is possible to calculate a hypothetical optimal frequency based on the fixed fiber threshold data for the BegoStone we tested and the fiber speed (Optimal frequency $=$ Fixed location stone volume $\mathrm{Hz}$ threshold $\times$ No. of distinct locations $\times$ fiber speed). For fiber speed of $1 \mathrm{~mm} /$ second, we calculated that 52 and 61.6 Hz was the frequency threshold for LP and MD modes, respectively. Increasing the fiber speed would increase these thresholds.

\section{Discussion}

In this in vitro study we investigated the effect of increasing pulse frequency to determine if there was a threshold for ablation and fragmentation efficiency during laser lithotripsy. First, we found that stone crater depth and volume increased significantly with increasing the number of pulses, but there was a threshold at $\sim 15$ pulses after which there was no significant increase in ablation. Second, lithotripsy efficiency and fragmentation rate was improved when the stone was broken down using a faster laser fiber speed. The impact of higher frequency on fragmentation is more pronounced when using a faster laser fiber speed. Third, the overall trend toward more fragmentation favored using pulse modulation with MD mode compared with LP, especially when the fiber was moved at $3 \mathrm{~mm} / \mathrm{second}$.

Data reported in our study are consistent with study presented recently by Chai and colleagues. ${ }^{13}$ They evaluated the effect of increasing the number of pulses on ablation in a single stone location. Using a BegoStone model, they assessed the effect of $40,80,120,240,360$, and 480 pulses on ablation crater volume. They demonstrated that 40 or 80 pulses could be the threshold after which increasing the number of pulses does not lead to an increase in ablation. In our study, we found the threshold is reached at a lower number of pulses compared with Chai and colleagues ${ }^{13}$ mainly because we started with lower number of pulses (1, $5,10,15,20,25,30,35$, and 40) compared with what they started with $(40,80,120,240,360$, and 480), which is already higher than the ablation threshold. Nonetheless, both studies demonstrate that there is a frequency threshold for ablation. 

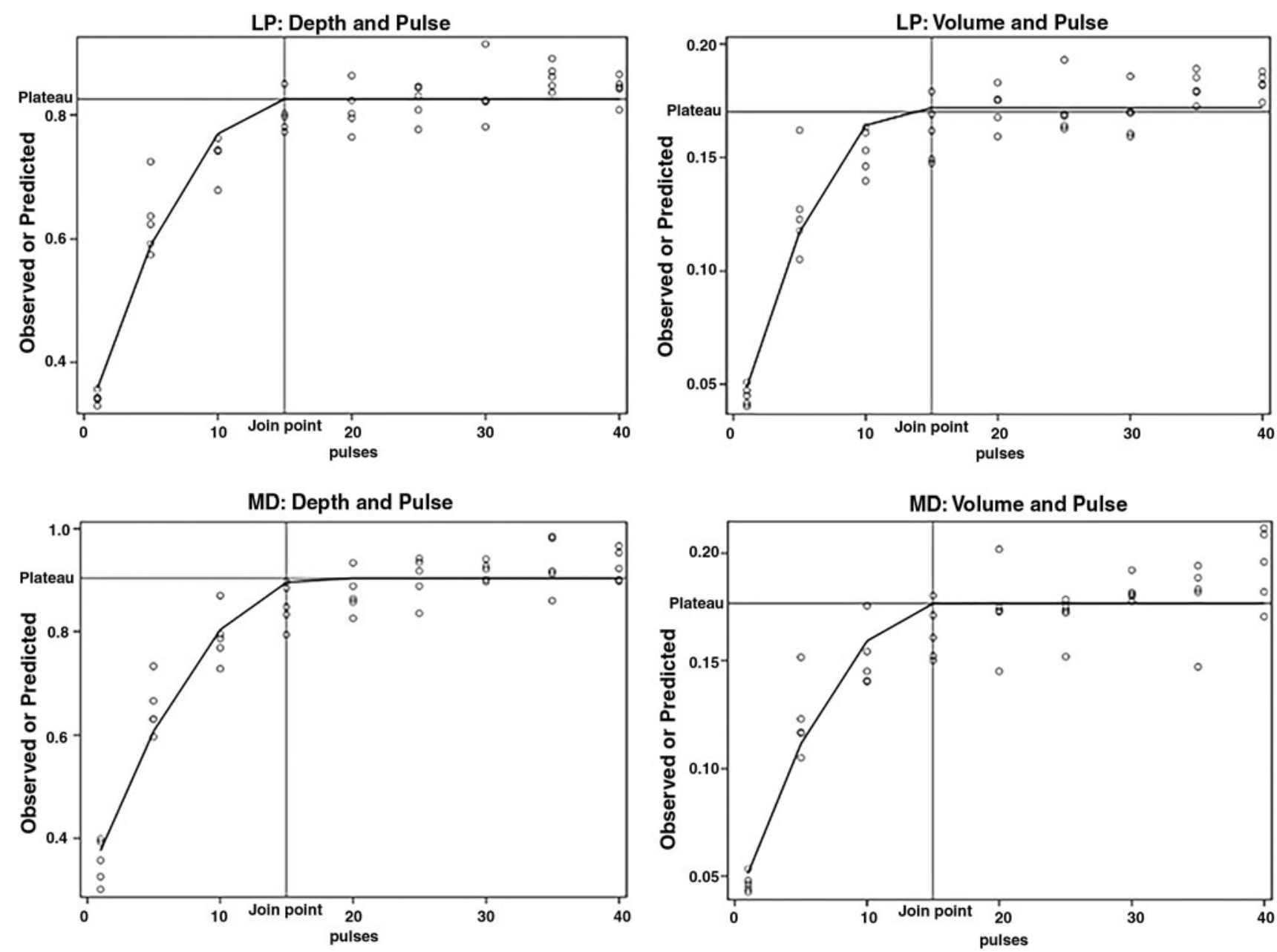

FIG. 2. Nonlinear segmented model demonstrating the relationship between the number of pulses, and crater depth and volume, for LP and MD modes.

Another study also supports the notion of a frequency threshold. Zhang and colleagues assessed ablation volume after delivering 1,3 , and 10 pulses to an artificial stone model. ${ }^{14}$ They also reported a nonlinear relationship between the pulse frequency and ablation volume.

Reduction in ablation per pulse with increasing number of pulses can be explained. Holmium laser energy is highly absorbed in water and the amount of energy travelling through fluid is reduced with distance. ${ }^{15}$ For stone ablation to occur, a direct contact between the laser fiber and the stone is needed to ensure direct absorption of the photons by the stone. ${ }^{16,17}$ As crater depth increases the amount of energy reaching the bottom of the crater reduces. The reduction in energy transmission results in reduction in ablation when the working distance between the fiber tip and the stone increases as reported recently. ${ }^{18-20}$ MD mode had statistically significant deeper craters than LP mode; however, this did not translate into larger ablation volumes when comparing MD with LP mode. This could be because of the wide variations in crater area and the small difference in crater depth between the two pulse modes, which was only $0.086 \mathrm{~mm}$.

We found that the speed of a moving laser fiber plays an important role in fragmentation. Exceeding the pulse fre- quency threshold at a single stone location results in minimal increase in ablation volume, wasted time, and wasted energy. Furthermore, dust ejecting from the crater after each pulse might have a shielding effect by absorbing some of the incoming laser energy during the subsequent pulses; thus, limiting the amount of energy reaching the stone. ${ }^{21}$ This further encourages continuous movement of the laser fiber in a painting technique. ${ }^{11}$ When the laser fiber was moved at $3 \mathrm{~mm} / \mathrm{second}$, the number of pulses delivered at each stone location was less compared with when the fiber was moving at $1 \mathrm{~mm} / \mathrm{second}$. Thus, an optimal number of pulses $(<15$ pulses) were delivered at each individual location as the fiber is moved, resulting in more fragmentation. In this regard our findings are new and can help better understand how pulse frequency affects fragmentation.

This study has several limitations. First, we used an artificial stone model made of BegoStone instead of human stones; the frequency threshold might differ for human kidney stones, and there may be variability between stone compositions. This difference is because of variations in crater volume produced for different stone compositions. ${ }^{22,23}$ We used BegoStone as this is the most commonly used model in lithotripsy research and provides consistent and 

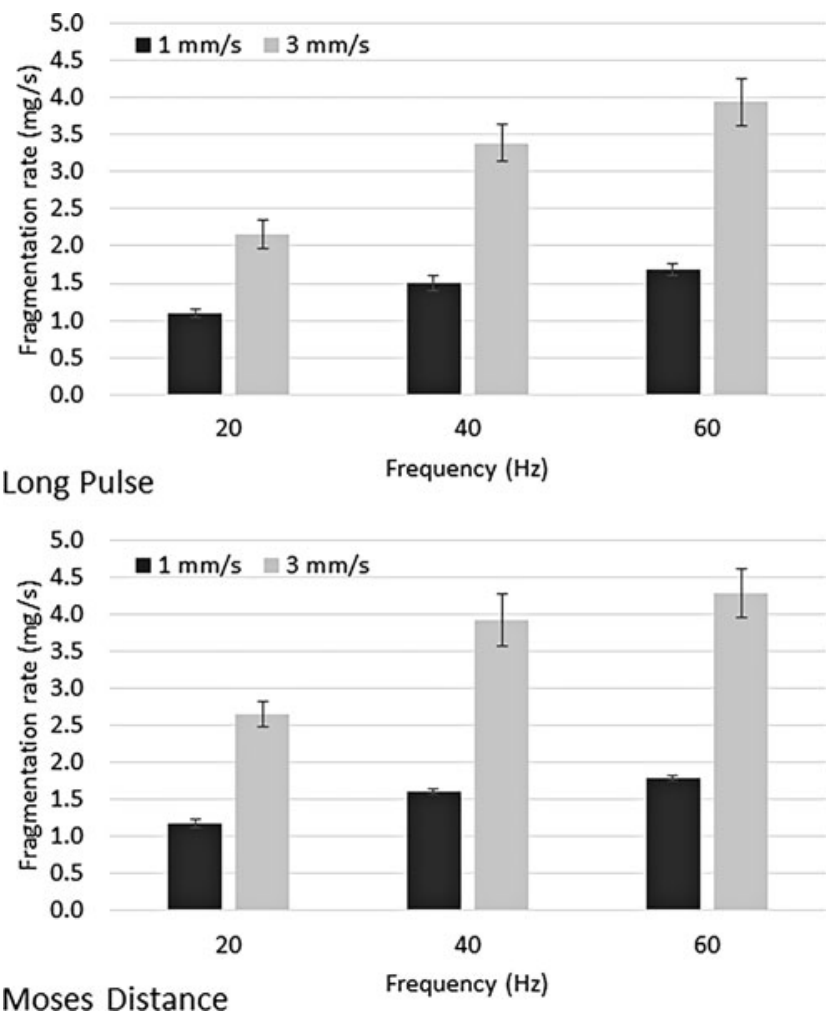

FIG. 3. Mean fragmentation rate for LP and MD modes at different pulse frequencies when the fiber was moving at 1 and $3 \mathrm{~mm} / \mathrm{second}$.

reproducible data. Also, we chose laser fiber movement speeds of 1 and $3 \mathrm{~mm} / \mathrm{second}$ when there is lack of clinical data to support any specific speed. However, in vitro videos of the laser fiber movement at these speeds were suggestive that $1 \mathrm{~mm} / \mathrm{second}$ is slower than what is observed clinically.

Another possible limitation in the moving fiber experiment is the possibility of 0.1 to $0.2 \mathrm{~mm}$ variations in fiber to stone distance when the fiber was moving. Keeping a $0 \mathrm{~mm}$ distance when the fiber is moving is technically challenging. To mitigate this we used a completely flat stone model as previously described, ${ }^{18}$ and although this is labor intensive, it is a critical step to get a completely flat stone surface, which if not done correctly can impact results

Table 1. Percentage Change in Fragmentation Rate for Different FreQuencies When the Fiber Was Moving at 1 AND $3 \mathrm{~mm} / \mathrm{SECOND}$

Frequency change Long pulse (\%) Moses distance (\%)

Percentage change at $1 \mathrm{~mm} /$ second

20 to $40 \quad 37.5 \quad 36.9$

40 to $60 \quad 11.2 \quad 11.1$

20 to $60 \quad 52.9 \quad 52.1$

Percentage change at $3 \mathrm{~mm} /$ second

20 to $40 \quad 56.5 \quad 48.1$

40 to $60 \quad 16.3 \quad 9.2$

20 to $60 \quad 82.1 \quad 61.7$

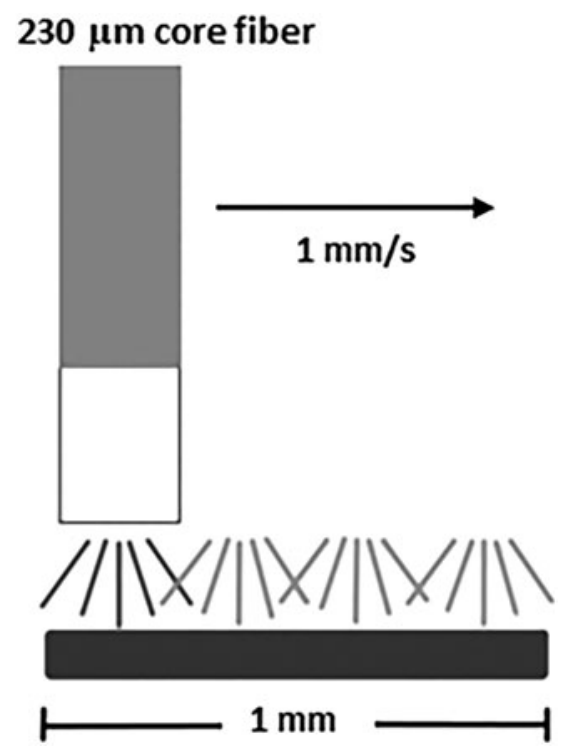

\section{$230 \mu \mathrm{m}$ core fiber}

FIG. 4. Schematic of a $230 \mu \mathrm{m}$ core laser fiber moving at $1 \mathrm{~mm} /$ second, illustrating the four distinct locations it would strike over a $1 \mathrm{~mm}$ stone distance.

based on fiber to stone distance. The use of our 3D positioner system is an important tool as it allows precise and consistent control in three axes. Keeping a fixed starting point for all experiments and repositioning the fiber before each line we kept the fiber to stone distance consistent for all experiments. Also, our experimental setup prevents stone retropulsion, which is an important outcome that can influence fragmentation in relation to frequency and stone movement.

In the fixed laser fiber experiments, pulses were delivered as single pulses and were separated in time. This method could limit any pulse to pulse effect that might occur if pulses were delivered in consecutively in 1 second. Although pulse system instability has been reported in older lasers, ${ }^{24,25}$ modern systems are built to provide more consistent performance. Controlling the number of pulses or sequence of pulses delivered would require access to the internal components of the laser system, which we could not do. However, delivering the pulses as single pulse ensured accuracy of the number of pulses delivered. In addition, stones were fixed in this study to limit stone retropulsion. Minimizing retropulsion was important in this study to limit its effect of study outcome. Finally, the formula we used to calculate the frequency needed might be an overestimate of the frequency when taking into consideration that the crater width generated from each pulse is larger than the core size of the laser fiber, but it provides a simple way to calculate how much frequency is needed. Further studies are needed to validate our findings.

Our study has a number of clinical implications. Our data demonstrated a nonlinear increase in ablation when increasing pulse frequency from 20 to 40 and 40 to $60 \mathrm{~Hz}$ when both laser fiber and stone movements were controlled. However, not all laser pulses will be delivered to the stone clinically because of stone and surgeon motion. The decision to increase pulse frequency should be balanced to ensure efficient utilization of energy delivered while moving the fiber in a painting technique during dusting. ${ }^{11}$ 
Furthermore, the speed of the fiber should be taken into consideration. Moving the fiber slowly reduces fragmentation rate and ultimately lithotripsy efficiency. Future research is needed to understand fiber movement speed; it is unclear how fast surgeons are moving the laser fiber during dusting laser lithotripsy. In particular, fiber speed has relevance for the development of robotic ureteroscopy platforms.

Our findings serve to optimize parameter selection and safety of laser lithotripsy, as well as inform the development of robotic ureteroscopy systems. Finally, by determining the frequency threshold for ablation, one can limit the pulse frequency to what would be most efficient. Reducing the frequency and, therefore, the total power during lithotripsy is of importance to limit the risk of thermal injury from heat generation. $^{26,27}$

In conclusion, the results of this in vitro study demonstrated that there is a frequency threshold after which increasing the pulse frequency leads to minimal gain in ablation when the laser fiber is fixed. When the fiber is moving, increasing the frequency increases fragmentation, but also only until a specific higher frequency threshold is reached. Our findings serve to provide more insight for parameter selection and safety of laser lithotripsy for the dusting technique.

\section{Acknowledgment}

We thank Yongmei Qin, biostatistician, for assistance with the statistical modeling.

\section{Author Disclosure Statement}

K.R.G. is a consultant for Lumenis and Boston Scientific. W.W.R. is a consultant for Boston Scientific. All other authors have no competing financial interests exist.

\section{Funding Information}

This study was supported by a scientific research grant from Boston Scientific.

\section{Supplementary Material}

Supplementary Figure S1

Supplementary Table S1

Supplementary Video S1

Supplementary Video S2

\section{References}

1. Tracey J, Gagin G, Morhardt D, Hollingsworth J, Ghani KR. Ureteroscopic high-frequency dusting utilizing a 120W holmium laser. J Endourol 2018;32:290-295.

2. Aldoukhi AH, Roberts WW, Hall TL, Ghani KR. Holmium laser lithotripsy in the new stone age: Dust or bust? Front Surg 2017;4:57.

3. Weiss B, Shah O. Evaluation of dusting versus basketingCan new technologies improve stone-free rates? Nat Rev Urol 2016;13:726-733.

4. Kronenberg $\mathrm{P}$, Traxer O. In vitro fragmentation efficiency of holmium: Yttrium-aluminum-garnet (YAG) laser lithotripsy-A comprehensive study encompassing different frequencies, pulse energies, total power levels and laser fibre diameters. BJU Int 2014;114:261-267.

5. Sea J, Jonat LM, Chew BH, et al. Optimal power settings for Holmium:YAG lithotripsy. J Urol 2012;187:914-919.

6. Vassar GJ, Teichman JM, Glickman RD. Holmium:YAG lithotripsy efficiency varies with energy density. J Urol 1998;160:471-476.

7. Wollin DA, Ackerman A, Yang C, et al. Variable pulse duration from a new holmium:YAG laser: The effect on stone comminution, fiber tip degradation, and retropulsion in a dusting model. Urology 2017;103:47-51.

8. Kuo RL, Aslan P, Zhong P, Preminger GM. Impact of holmium laser settings and fiber diameter on stone fragmentation and endoscope deflection. J Endourol 1998;12: 523-527.

9. Ritchie C, Yang P, Peplinski B, et al. Jackets off: The impact of laser fiber stripping on power output and stone degradation. J Endourol 2017;31:780-785.

10. Vassantachart JM, Lightfoot M, Yeo A, et al. Laser fiber cleaving techniques: Effects on tip morphology and power output. J Endourol 2015;29:84-89.

11. Hecht SL, Wolf JS, Jr. Techniques for holmium laser lithotripsy of intrarenal calculi. Urology 2013;81:442445.

12. Aldoukhi AH, Black KM, Hall TL, et al. Defining thermally safe laser lithotripsy power and irrigation parameters: In vitro model. J Endourol 2020;34:76-81.

13. Chai D, Zhang JJ, Podana N, Xuan RJ, Hasenberg T, Harrah T. The study of Ho:YAG laser ablation thresholds of calculus phantom in terms of peak power density (conference presentation). San Francisco: SPIE, 2019.

14. Zhang JJ, Rutherford J, Solomon M, et al. Numerical response surfaces of volume of ablation and retropulsion amplitude by settings of Ho:YAG laser lithotripter. J Healthc Eng 2018;2018:8261801.

15. van Leeuwen TG, van der Veen MJ, Verdaasdonk RM, Borst C. Noncontact tissue ablation by holmium: YSGG laser pulses in blood. Lasers Surg Med 1991;11: 26-34.

16. Jansen ED, van Leeuwen TG, Motamedi M, Borst C, Welch AJ. Temperature dependence of the absorption coefficient of water for midinfrared laser radiation. Lasers Surg Med 1994;14:258-268.

17. Vassar GJ, Chan KF, Teichman JM, et al. Holmium:YAG lithotripsy: Photothermal mechanism. J Endourol 1999;13: 181-190.

18. Aldoukhi AH, Roberts WW, Hall TL, Ghani KR. Watch your distance: The role of laser fiber working distance on fragmentation when altering pulse width or modulation. J Endourol 2019;33:120-126.

19. Winship B, Wollin D, Carlos E, et al. Dusting efficiency of the Moses holmium laser: An automated in vitro assessment. J Endourol 2018;32:1131-1135.

20. De Coninck V, Keller EX, Chiron P, et al. Ho:YAG laser lithotripsy in non-contact mode: Optimization of fiber to stone working distance to improve ablation efficiency. World J Urol 2019;37:1933-1939.

21. Randad A, Ahn J, Bailey MR, et al. The impact of dust and confinement on fragmentation of kidney stones by shockwave lithotripsy in tissue phantoms. J Endourol 2019;33: 400-406.

22. Chan KF, Hammer DX, Choi B, et al. Free electron laser lithotripsy: Threshold radiant exposures. J Endourol 2000; 14:161-167. 
23. Teichman JM, Vassar GJ, Glickman RD. Holmium: yttrium-aluminum-garnet lithotripsy efficiency varies with stone composition. Urology 1998;52:392-397.

24. Bader MJ, Pongratz T, Khoder W, et al. Impact of pulse duration on Ho:YAG laser lithotripsy: Fragmentation and dusting performance. World J Urol 2015;33:471477.

25. Lee H, Kang HW, Teichman JM, Oh J, Welch AJ. Urinary calculus fragmentation during Ho:YAG and Er:YAG lithotripsy. Lasers Surg Med 2006;38:39-51.

26. Aldoukhi AH, Hall TL, Ghani KR, Maxwell AD, MacConaghy $\mathrm{B}$, Roberts WW. Caliceal fluid temperature during high-power holmium laser lithotripsy in an in vivo porcine model. J Endourol 2018;32:724-729.

27. Wang XK, Jiang ZQ, Tan J, Yin GM, Huang K. Thermal effect of holmium laser lithotripsy under ureteroscopy. Chin Med J (Engl) 2019;132:2004-2007.
Address correspondence to: Ali H. Aldoukhi, MBBS, MS Department of Urology

University of Michigan Medical Sciences Unit I, Room 4432

1301 Catherine Street Ann Arbor, MI, 48109-2900

USA

E-mail: adoukhi@bu.edu

\author{
Abbreviations Used \\ $3 \mathrm{D}=$ three-dimensional \\ $\mathrm{LP}=$ long pulse \\ $\mathrm{MD}=$ Moses distance
}

